\section{Should IV Antibiotics Be Administered by Prolonged Infusion?}

\section{THE “PRO” SIDE}

Prolonged infusion of antimicrobial agents has been suggested as a means of optimizing therapy for infectious diseases. Opponents of this approach claim a lack of clinical evidence and the need for extensive resources to support these activities. Our position is that prolonged infusion of antimicrobials can save lives. Our focus is on serious infections and the use of time-dependent agents like the penicillins and carbapenems.

We advocate prolonged infusions to combat the potential misuse of time-dependent antimicrobials, driven by the following myths:

- A laboratory-reported "S" (for "susceptible") means that the agent will be effective, and all S's are equal.

- Manufacturer-recommended doses are always more than is necessary.

- If treatment failure occurs, it must be due to factors other than the antimicrobial agent.

- Resistance is "inevitable", and discovering new agents is the only solution.

Prolonged infusions of time-dependent antibiotics maximize the achievement of relevant therapeutic concentrations over time (i.e., pharmacokinetics) and allow for maximum action of the drug (i.e., pharmacodynamics). The pharmacokinetics and pharmacodynamics (PK/PD) of antimicrobials form the foundation of drug dose development to optimize clinical outcomes. ${ }^{1}$ PK/PD are essential in the study of new agents to determine best dosing regimens and to establish microbiological breakpoints for susceptibility. ${ }^{2.3}$ For existing antimicrobials, PK/PD are used to investigate the adequacy of traditional dosing in relation to clinical efficacy and emergence of resistance. ${ }^{4,5}$ Once established within clinical trials, $\mathrm{PK} / \mathrm{PD}$ principles provide valuable information for further exploration. For infectious diseases, the trials otherwise needed to study each permutation and combination of patient population, site of infection, and pathogen would be innumerable.

For time-dependent antimicrobials, the percentage of time that free concentrations exceed the minimum inhibitory concentration ( $\% f \mathrm{~T}_{>_{\text {MIC }}}$ ) is the most relevant surrogate for clinical outcome. Published targets vary depending on drug class (e.g., penicillins or carbapenems), study design (e.g., in vitro or in vivo), and the response measured, such as microbiologic activity (e.g., bacteriostatic or bactericidal) or clinical outcome (e.g., cure or survival). There are important considerations when applying PK/PD targets in the clinical setting. Although thresholds of $40 \%-50 \% f \mathrm{~T}_{\text {>MIC }_{\text {IC }}}$ have been found to be significant and have been widely adopted, they may not be optimal in all cases.
Recent clinical evidence, for example, has shown even better outcomes for patients with serious infections when higher targets, for example, above $75 \% f \mathrm{~T}_{\text {>MIC }}$, are achieved. ${ }^{4,6-8}$ It is our premise in this article that optimal targets should be sought, rather than just exceeding minimum thresholds such as the 20\% for carbapenems, $30 \%$ for penicillins, or $40 \%$ for cephalosporins frequently cited in the literature. ${ }^{9}$

Standard recommended doses are largely based on the "average" or "typical" patient, with little guidance for dose individualization. The limitations of standard doses for timedependent antimicrobials include the following:

- narrow range in dosing options (e.g., 1-2 g q12h)

- lack of weight-based dosing, which assumes a uniform drug distribution space (also known as volume of distribution) for all patients

- no adjustment for very high creatinine clearance, as seen with hyperfiltration in critically ill patients

- simple doubling of the dose for serious infections (e.g., cefotaxime from $1 \mathrm{~g}$ to $2 \mathrm{~g} \mathrm{q} 8 \mathrm{~h}$ ) has minimal effect on $\% f \mathrm{~T}_{\text {MIC }}$ for common ß-lactams, which have very short half-lives

- no consideration for case-specific microbiology or local pathogen susceptibilities

Standard recommended doses cannot meet the needs of all patients. The same PK/PD principles that are used to generate regimens for the "average" patient can now be used to determine dosing for those at high risk of antimicrobial failure. These patients may include people with significantly altered pharmacokinetics (e.g., because of obesity, critical illness, or burns), immunosuppression (e.g., with diabetes mellitus or neutropenia), or less susceptible pathogens (e.g., Pseudomonas aeruginosa). In the above cases, prolonged infusions of timedependent antimicrobials over 2 to $4 \mathrm{~h}$ can achieve PK/PD targets not attained by standard administration. Any concerns about logistical barriers and the resources needed to administer prolonged infusions of antimicrobials are outweighed by the potential life-saving benefits of individualized therapy.

As just one example, the advantages of prolonged infusion have become evident for piperacillin-tazobactam, an extendedspectrum penicillin widely used in the treatment of serious infections, such as intra-abdominal sepsis and nosocomial pneumonia. As we have shown using Monte Carlo simulation, prolonged administration can significantly improve the $\mathrm{PK} / \mathrm{PD}$ performance of this antimicrobial, which is especially desirable for the critically ill population. Monte Carlo simulation is a robust research tool that is extensively used in engineering, computer sciences, finance, and, more recently, the biomedical sciences. In the area of antimicrobial PK/PD, which has numerous confounding variables, Monte Carlo simulation can be applied to evaluate dosing regimens in large numbers of simulat- 
ed patients based on specific demographics, antimicrobial $\mathrm{PK} / \mathrm{PD}$, and pathogen susceptibilities. Instead of defaulting to "average" patients or worst-case scenarios, practitioners can use the results of Monte Carlo simulations that are relevant to the patient populations of interest.

Using Monte Carlo simulation, we studied empiric antimicrobial therapies for intensive care unit (ICU) patients with infection..$^{10}$ Serum concentration profiles were constructed from population pharmacokinetic models. MIC distributions were obtained from a Canadian surveillance network tracking 4798 ICU pathogens collected from 2005 to 2008. ${ }^{11}$ For time-dependent agents, $>75 \% f \mathrm{~T}_{\text {MIC }}$ was selected as the optimal clinical target for antimicrobial therapy. We found that standard piperacillin-tazobactam dosing with $3.375 \mathrm{~g}$ q6h (0.5-h infusion) would achieve the target in just $60 \%$ of cases, whereas simply prolonging the infusion to $3 \mathrm{~h}$ would allow the threshold to be reached in $79 \%$ of the population. Figure 1 shows the mean concentrations for those regimens relative to the susceptible MIC breakpoint of $16 \mu \mathrm{g} / \mathrm{mL}$ for Enterobacteriaceae set by the Clinical Laboratory Standards Institute. It is clear from this graph that for the same dose of piperacillin-tazobactam, prolonged infusion would significantly extend the $\% f \mathrm{~T}_{\text {MIC }}$ relative to the standard infusion. Further analysis showed that increasing the dose to $4.5 \mathrm{~g}$ q6h (0.5-h infusion) would have limited impact, such that the target would be reached in only $65 \%$ of the study population.

Lodise and others ${ }^{12}$ recently demonstrated the clinical benefits of prolonged infusion of piperacillin-tazobactam. In a study of 194 patients receiving piperacillin-tazobactam for infection with susceptible $P$. aeruginosa, mortality rates were considerably lower among those who received prolonged infusions than among those who received standard intermittent doses $(12.2 \%$ versus $31.6 \%, p=0.04)$. Our Monte Carlo simulations support these findings, showing that traditional regimens would be unable to achieve reasonable PK/PD targets against $P$. aeruginosa. ${ }^{10}$ Even at $4.5 \mathrm{~g}$ q6h (0.5-h infusion), the target was attained in only $39 \%$ of cases, whereas prolonged infusion over $3 \mathrm{~h}$ led to target attainment in close to $70 \%$ of the study population.

There have been longstanding concerns regarding the adequacy of piperacillin-tazobactam for treating pseudomonal infections. ${ }^{13}$ In fact, for nosocomial pneumonia the current product monograph advises piperacillin-tazobactam $4.5 \mathrm{~g}$ q6h, in combination with an aminoglycoside. ${ }^{14}$ The rationale for such concern and aggressive therapy are consistent with our argument of poor target attainment with standard recommended doses. However, as shown earlier, the PK/PD advantages of increasing the dose are minimal compared with those of other strategies such as prolonged infusion.

Similar benefits may be observed with other timedependent antimicrobials, especially those with short half-lives. One example is meropenem, for which prolonged infusions can beneficially extend $\% f \mathrm{~T}_{\text {MIC }_{\mathrm{C}}}$ beyond critical targets. Antimicrobials are commonly studied in noninferiority trials with highly selected populations, and, as previously discussed,

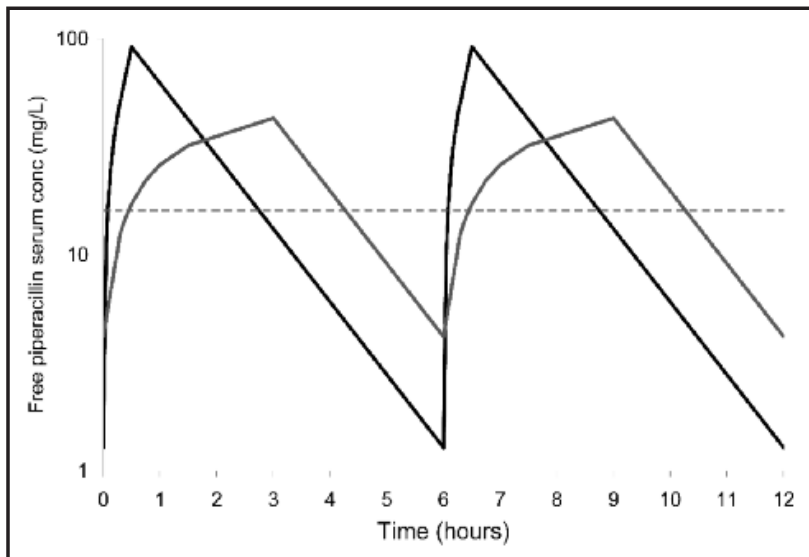

Figure 1. Mean piperacillin concentrations based on Monte Carlo simulation of 5000 patients in the intensive care unit receiving $3.375 \mathrm{~g}$ q6h with 0.5-h infusion (solid black line) or $3.375 \mathrm{~g}$ q6h with 3-h infusion (solid grey line). Hatched grey line indicates the sensitive minimum inhibitory concentration break point $(16 \mu \mathrm{g} / \mathrm{L})$ for Enterobacteriaceae.

standard recommended doses do not ensure the best outcome for all patients. Prolonged infusion is one method of adjusting drug dosing for those who do not fit the "average" patient profile. The challenge for clinicians is to identify patients most likely to derive significant benefit from dose individualization, such as those who are infected with less susceptible pathogens, obese, immunocompromised, critically ill, or in septic shock. For time-dependent antimicrobials, prolonged infusion, along with other strategies such as shortened dosing interval or continuous infusion, can be used to overcome the inadequacy of coverage provided by standard intermittent regimens.

\section{References}

1. Drusano GL. Antimicrobial pharmacodynamics: critical interactions of 'bug and drug.' Nat Rev Microbiol 2004;2(4):289-300.

2. Drusano GL, Preston SL, Hardalo C, Hare R, Banfield C, Andes D, et al. Use of preclinical data for selection of a phase II/III dose for evernimicin and identification of a preclinical MIC breakpoint. Antimicrob Agents Chemother 2001;45(1):13-22.

3. Mouton J, Schmitt-Hoffmann A, Shapiro S, Nashed N, Punt NC. Use of Monte Carlo simulations to select therapeutic doses and provisional breakpoints of BAL9141. Antimicrob Agents Chemother 2004; 48(5):1713-1718.

4. Ariano RE, Nyhlén A, Donnelly JP, Sitar DS, Harding GK, Zelenitsky SA. Pharmacokinetics and pharmacodynamics of meropenem in febrile neutropenic patients with bacteremia. Ann Pharmacother 2005;39(1): 32-38.

5. Zelenitsky S, Ariano R, Harding G, Forrest A. Evaluating ciprofloxacin dosing for Pseudomonas aeruginosa infection by using clinical outcomebased Monte Carlo simulations. Antimicrob Agents Chemother 2005;49(1):4009-4014.

6. Li C, Du X, Kuti JL, Nicolau DP. Clinical pharmacodynamics of meropenem in patients with lower respiratory tract infections. Antimicrob Agents Chemother 2007;51:1725-1730.

7. McKinnon PS, Paladino JA, Schentag JJ. Evaluation of area under the inhibitory curve (AUIC) and time above the minimum inhibitory concentration ( $>$ MIC) as predictors of outcome for cefepime and ceftazidime in serious bacterial infections. Int $J$ Antimicrob Agents 2008;31(4):345-351.

8. Tam VH, McKinnon PS, Akins RL, Rybak MJ, Drusano GL. Pharmacodynamics of cefepime in patients with Gram-negative infections. 
I Antimicrob Chemother 2002;50(3):425-428.

9. Lodise T, Lomaestro BM, Drusano GL. Application of antimicrobial pharmacodynamic concepts into clinical practice: focus on beta-lactam antibiotics. Pharmacotherapy 2006;26(9):1320-1332.

10. Zelenitsky S, Ariano RE, Zhanel GG. Characterizing infections and appropriate antibiotic use in Canadian intensive care units (ICUs) using national surveillance data from 2005-2008 [slide session 0-1789]. In: 49th Interscience Conference on Antimicrobial Agents and Chemotherapy (ICAAC); 2009 Sep 14; San Francisco (CA).

11. Zhanel GG, DeCorby M, Laing N, Weshnoweski B, Vashisht R, Tailor $\mathrm{F}$, et al. Antimicrobial-resistant pathogens in intensive care units in Canada: results of the Canadian National Intensive Care Unit (CAN-ICU) Study, 2005-2006. Antimicrob Agents Chemother 2008; 52(4):1430-1437.

12. Lodise TP Jr, Lomaestro B, Drusano GL. Piperacillin-tazobactam for Pseudomonas aeruginosa infection: clinical implications of an extendedinfusion dosing strategy. Clin Infect Dis 2007;44(3):357-363.

13. A reminder: piperacillin/tazobactam is not for Pseudomonas. Med Lett 1984;36:110.

14. Tazocin: sterile piperacillin sodium/tazobactam sodium. Lyophilized powder for injection. For intravenous use [product monograph]. St Laurent (QC): Wyeth Canada; 2008. In: Drug Product Database online query. Ottawa (ON): Health Canada [cited 2010 May 18]. Available from: http://webprod.hc-sc.gc.ca/dpd-bdpp/index-eng.jsp

Robert Ariano, PharmD, BCPS, FCCM

Pharmacy Department

St Boniface General Hospital

Winnipeg, Manitoba

Sheryl Zelenitsky, BScPharm, PharmD

Faculty of Pharmacy

University of Manitoba

Winnipeg, Manitoba

\section{THE "CON" SIDE}

I was challenged by the editors of this journal to "author the con side" of this question and to "angle it however [I] choose." Further guidelines were that this was not intended to be a "discussion on continuous infusion of antibiotics, but rather the 'cons' of several-hour intermittent bolus infusions versus 30- to 60-minute infusions."

So, rather than a somewhat selective presentation of the evidence to support the "con" side, my so-called angle for this piece is to present what I hope will be a cogent synopsis and critique of the relevant evidence on the issue of extended infusion of antibiotics. I believe the only definitive way to figure out if one way of delivering antibiotics improves outcomes better than another way is to randomly assign patients who require parenteral antibiotics to receive the agent by the different delivery methods and see what happens. Although the pharmacokinetic-pharmacodynamic (PK/PD) literature is certainly useful for developing hypotheses, infectious disease practice in particular is fraught with concepts that either have fallen by the wayside (e.g., serum bactericidal titers) or should have fallen by the wayside (e.g., measuring aminoglycoside levels ${ }^{1,2}$ ) because of a lack of solid clinical evidence.
So, given the above challenge and my belief structure, I did what I think any reasonable person with an "evidence-based" mindset would do: I searched a number of bibliographic databases, including MEDLINE and the Cochrane Library, as well as using Google to search the Internet. Lo and behold, I found a recent, well-done systematic review with the objective of determining "whether any clinical benefits exist for administration of B-lactam antibiotics by extended or continuous infusion". 3 Does life get any better than that?

Roberts and others ${ }^{3}$ reviewed the medical literature up to November 2007 and found 14 randomized controlled trials (755 patients in total) that could be incorporated into a meta-analysis. Their overall findings were as follows: "The use of continuous infusion or extended administration of B-lactam was not associated with a significant improvement in the clinical cure (odds ratio 1.04) or mortality (odds ratio 1.00)." These fairly definitive results indicate that how you infuse ß-lactams doesn't make a difference clinically.

Perhaps of more relevance to this particular debate is that in only 1 of the 14 trials was extended administration (in contrast to continuous infusion) actually evaluated, and that study ${ }^{4}$ had only 5 patients in each arm. Not surprisingly, a difference wasn't found.

Roberts and others ${ }^{3}$ also reported on 2 observational studies, one of which examined extended infusions. Lodise and others ${ }^{5}$ reported a retrospective study of 194 critically ill patients, 92 of whom received regular infusions and 102 of whom, following a change in practice, received extended (i.e., 4-h) infusions of piperacillin-tazobactam. The mortality rate was lower, with an absolute difference of $20 \%$ (12\% versus $32 \%$ ), and hospital stays were shorter for patients who received the extended infusions. Interestingly, the reason the cohort was evaluated this way was a previous Monte Carlo "mathematical simulation" performed by the authors, which was "so compelling that the novel extendedinfusion protocol was quickly adopted into practice"; this precluded a better-designed comparison of the 2 infusion methods. Nonetheless, results of this magnitude, if real, would of course have great importance. However, the same investigators ${ }^{6}$ more recently reported a second retrospective study, also with piperacillin-tazobactam, of 129 patients with Gram-negative infections. In that investigation, they found no difference between the 2 cohorts in terms of either mortality or length of stay.

The final place I looked was clinicaltrials.gov, where I found reference to an ongoing trial in the intensive care setting (NCT00891423; estimated completion date January 2010) in which meropenem was being given by infusion over either 30 min (1-g dose) or $3 \mathrm{~h}$ (500-mg dose) to patients with a serious infection. Unfortunately, the estimated enrolment was 10 patients, so I'm not sure how useful this evidence will be.

Now, there appear to be a fair number of PK/PD publications (the latter reporting on surrogate markers, not clinical outcomes) suggesting a theoretical benefit of continuous antibiotic infusions based on concepts like time above the minimum inhibitory concentration and other theoretical 
concepts. In fact, a very recent one was published by the authors of the "pro" side of this debate. Nicasio and others" did a good job of showing "that cefepime dosed $2 \mathrm{~g}$ every $8 \mathrm{~h}$ as a 3-h prolonged infusion will improve the likelihood of pharmacodynamic target attainment over that of standard 30-min infusions", but the clinical relevance of this and, in fact, all the other $\mathrm{PK} / \mathrm{PD}$ evaluations is unknown.

So, overall there are 3 publications (a 10-patient randomized controlled trial and 2 retrospective evaluations, one showing a benefit and the other no benefit) and one 10-subject study-inprogress looking at clinically relevant end points associated with extended infusion of antibiotics. Lastly, a 2009 editorial in reference to the above-mentioned meta-analysis ${ }^{3}$ was entitled "The jury is still out on continuous infusion of B-lactam antibiotics in intensive care patients" . ${ }^{8}$ However, in my mind, when it comes to the issue of extended infusions, the case wouldn't even have gone to trial, let alone a juried trial; it should have been thrown out of court for lack of evidence!

On the face of it, do I think that changing how you infuse an antibiotic will improve outcomes? No-there are far too many factors influencing the outcome of infectious diseases, only one of which is the delivery rate of antibiotics. Nonetheless, I would love the advocates of this approach to prove me wrong by conducting well-designed clinical trials to definitively answer the question.

Now, one can probably conclude, on the basis of the available evidence, that an extended infusion is no better or worse than any other method of delivering an antibiotic. So, if there are economic advantages, as some suggest, ${ }^{9}$ or convenience reasons for delivering an antibiotic this way, then great . . . go ahead and do it. However, there are a few issues related to the use of extended infusions that should be considered:

- Some B-lactams may not be stable for long enough to allow extended infusion.

- Additional lines may be needed to prevent incompatibilities with other drugs.

- The presence of an infusion pump limits patient mobility.

So what should clinicians do to best improve how they dose antibiotics? Well, when I was a "baby" PharmD student at the
Medical University of South Carolina in the mid-1980s, I was following a patient with Pseudomonas osteomyelitis of the jawbone. I remember asking the attending infectious diseases physician how he figured out the appropriate dose of ceftazidime to use. He looked me in the eye, and said (add a bit of a southern drawl for effect), "Well, let me tell you; 1 gram feels good, but 2 grams feels real good!" Since that time, I haven't heard any better advice or seen any evidence that improves on that concept.

\section{References}

1. McCormack JP, Jewesson PJ. A critical reevaluation of the "therapeutic range" of aminoglycosides. Clin Infect Dis 1992;14(1):320-339.

2. McCormack JP. An emotional-based medicine approach to monitoring once-daily aminoglycosides. Pharmacotherapy 2000;20(12):1524-1527.

3. Roberts JA, Webb S, Paterson D, Ho KM, Lipman J. A systematic review on clinical benefits of continuous administration of B-lactam antibiotics. Crit Care Med 2009;37(6):2071-2078.

4. Kojika M, Sato N, Hakozaki M, Suzuki Y, Takahasi G, Endo S, et al. [A preliminary study of the administration of carbapenem antibiotics in sepsis patients on the basis of the administration time]. Jpn J Antibiot 2005;58(5):452-457. Japanese.

5. Lodise TP Jr, Lomaestro B, Drusano GL. Piperacillin-tazobactam for Pseudomonas aeruginosa infection: clinical implications of an extendedinfusion dosing strategy. Clin Infect Dis 2007;44(3):357-363.

6. Patel GW, Patel N, Lat A, Trombley K, Enbawe S, Manor K, et al. Outcomes of extended infusion piperacillin/tazobactam for documented Gram-negative infections. Diagn Microbiol Infect Dis 2009; 64(2):236-240.

7. Nicasio AM, Ariano RE, Zelenitsky SA, Kim A, Crandon JL, Kuti JL, et al. Population pharmacokinetics of high-dose, prolonged-infusion cefepime in adult critically ill patients with ventilator-associated pneumonia. Antimicrob Agents Chemother 2009;53(4):1476-1481.

8. van Zanten AR. The jury is still out on continuous infusion of betalactam antibiotics in intensive care patients. Crit Care Med 2009;37(6):2137-2138.

9. van Zanten AR, Engelfriet PM, van Dillen K, van Veen M, Nuijten MJ, Polderman KH. Importance of nondrug costs of intra venous antibiotic therapy. Crit Care 2003;7(6):R184-R190.

James McCormack, BSc(Pharm), PharmD

Faculty of Pharmaceutical Sciences

University of British Columbia

Vancouver, British Columbia 